This item was submitted to Loughborough's Research Repository by the author.

Items in Figshare are protected by copyright, with all rights reserved, unless otherwise indicated.

\title{
Optimization of the high-frequency torsional vibration of vehicle driveline systems using genetic algorithms
}

\section{PLEASE CITE THE PUBLISHED VERSION}

\section{PUBLISHER}

Professional Engineering Publishing / @ IMECHE

\section{VERSION}

VoR (Version of Record)

\section{LICENCE}

CC BY-NC-ND 4.0

\section{REPOSITORY RECORD}

Farshidianfar, A., M. Ebrahimi, Homer Rahnejat, M.T. Menday, and M. Moavenian. 2019. "Optimization of the High-frequency Torsional Vibration of Vehicle Driveline Systems Using Genetic Algorithms". figshare. https://hdl.handle.net/2134/4791. 
This item was submitted to Loughborough's Institutional Repository (https://dspace.lboro.ac.uk/) by the author and is made available under the following Creative Commons Licence conditions.

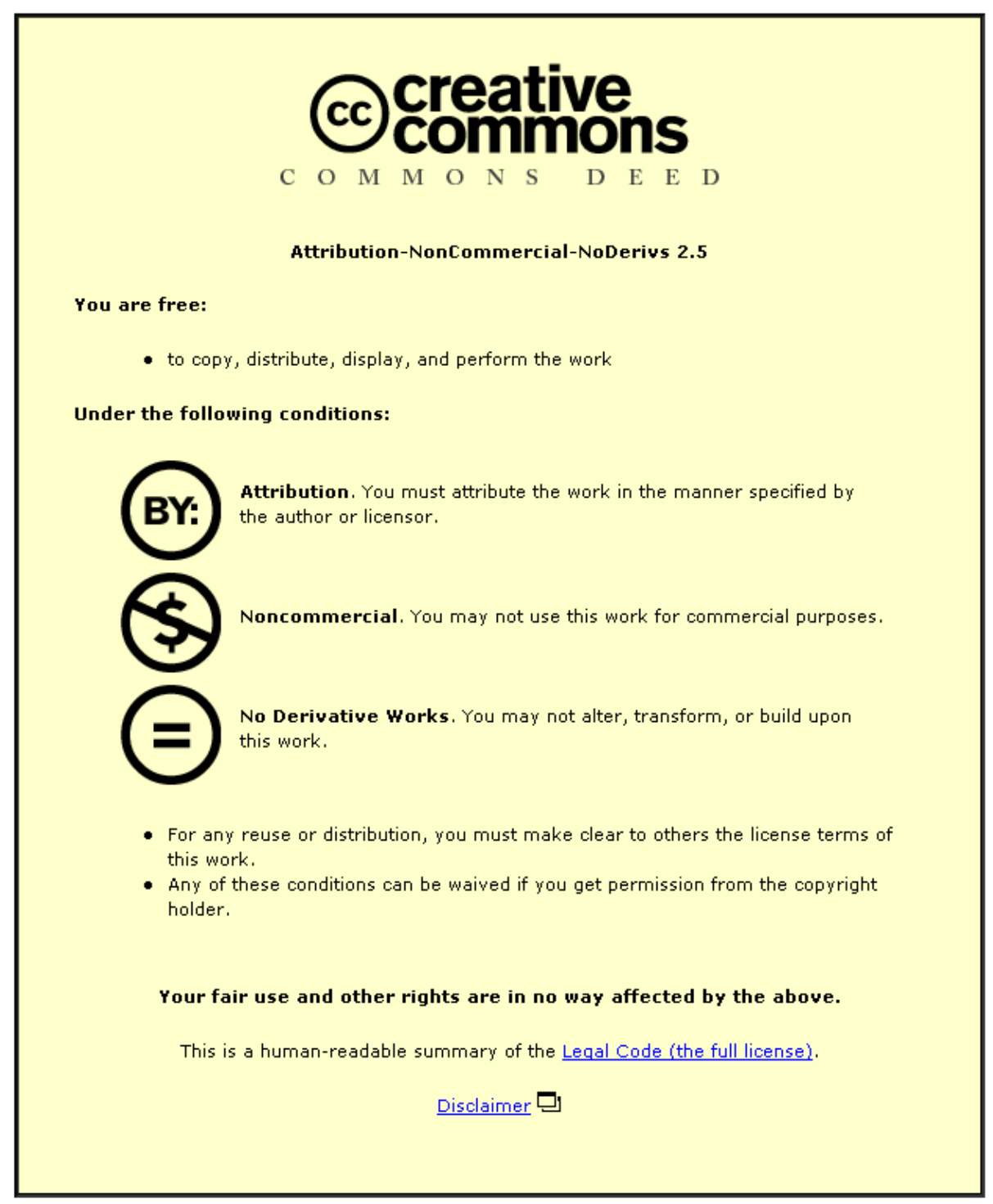

For the full text of this licence, please go to: http://creativecommons.org/licenses/by-nc-nd/2.5/ 


\title{
Optimization of the high-frequency torsional vibration of vehicle driveline systems using genetic algorithms
}

\author{
A Farshidianfar ${ }^{1}, \mathbf{M}$ Ebrahimi ${ }^{1}, \mathbf{H}$ Rahnejat $^{2} *, \mathbf{M} \mathbf{T ~ M e n d a y ~}^{3}$ and $\mathbf{M}$ Moavenian $^{4}$ \\ ${ }^{1}$ Department of Mechanical and Medical Engineering, University of Bradford, UK \\ ${ }^{2}$ Wolfson School of Mechanical and Manufacturing Engineering, Loughborough University, UK \\ ${ }^{3}$ Powertrain Systems Engineering, Ford Engineering Research Centre, Dunton, UK \\ ${ }^{4}$ Department of Mechanical Engineering, University of Ferdowsi, Mashhad, Iran
}

\begin{abstract}
Vehicle drivelines with manual transmissions are exposed to different dynamic engine torques under driving conditions. Engine torque can dramatically vary with throttle demand from coast to drive condition and, conversely, with throttle release from drive to coast. Abrupt application or release of throttle in slow moving traffic or rapid engagement of the clutch can be followed by an audible response, referred to in industry as the clonk noise. This paper presents a complete dynamic model of a vehicle driveline for the optimization of high-frequency torsional vibration by the distributed-lumped (hybrid) modelling technique (DLMT). The model used is first validated against experimental tests. Parameter sensitivity studies have been carried out using the model to identify the important components affecting clonk. Three key parameters have been chosen from the parameter study. To optimize these key factors, genetic algorithms (GAs) have been used in this multi-parameter optimization problem. The GAs show significant reduction in the driveline noise, vibration and harshness (NVH).
\end{abstract}

Keywords: driveline NVH, shuffle, clonk distributed-lumped modelling, high-frequency vibration response, genetic algorithms

\section{NOTATION}

$B_{\mathrm{f}}, B_{\mathrm{g}}, B_{\mathrm{d}}, B_{\mathrm{w}}$

equivalent coefficients of viscous

damping

$C_{\mathrm{c}}, C_{\mathrm{p}}, C_{\mathrm{a}}$

$C_{j}$

$E_{j}$

$G_{1}, G_{2}$

$J_{\mathrm{f}}, J_{\mathrm{g}}, J_{\mathrm{d}}, J_{1}$, $J_{2}, J_{3}, J_{\mathrm{w}}$ $k_{\mathrm{c}}, k_{1}, k_{2}, k_{\mathrm{p}}$

$l_{j}$

$L_{j}$

$n_{\mathrm{d}}$

$n_{\mathrm{g}}$

$s$ coefficients of viscous damping for the

clutch

shaft compliance per unit

length $=1 / G J(j=i$ th element -1$)$

frequency transform variable $(j=i$ th

element - 1)

shear modulus of rigidity

second polar moments of inertia

torsional stiffnesses

shaft lengths $(j=i$ th element -1$)$

shaft inertia per unit length $=J \rho$

$(j=i$ th element -1$)$

differential gear ratio

gearbox gear ratio

Laplace variable

The MS was received on 7 June 2001 and was accepted after revision for publication on 28 March 2002.

*Corresponding author: Wolfson School of Mechanical and Manufacturing Engineering, University of Loughborough, Loughborough, Leicatershire LEII 3TU, UK

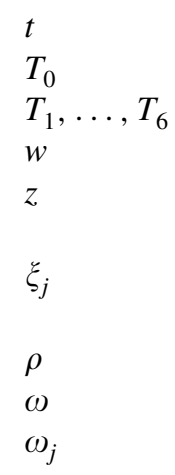

time

input torque

transmitted torques

transform variable

finite time delay

characteristic impedances $(j=i$ th element -1 ) material density frequency angular velocities $(j=i$ th element -1 )

\section{INTRODUCTION}

The vehicular driveline system comprises engine, clutch, transmission system, driveshaft tubes, differential, rear axle half-shafts and rear road wheels. This is a complex assembly of active and reactive dynamic elements. The driveline is highly non-linear and lightly damped, and thus readily excited by engine and road inputs. The driveline is a source of noise, vibration and harshness $(\mathrm{NVH})$ concern with a large spectrum of response frequencies. A typical driveline NVH spectrum contains a significant number of frequencies, 
ranging from a few $\mathrm{Hz}$ to several $\mathrm{kHz}$ [1]. This paper is concerned with high-frequency structural-acoustic response, commonly referred to as driveline clonk, a short-duration, audible, high-frequency transient response. This occurs as a result of load reversals in the presence of lashes in the system. The load reversal is usually caused by torsional impact of contacting elements by take-up of backlash, for example in geared transmissions, spline joints or at the differential. Load reversal can also manifest itself through low-frequency torsional vehicle driveline shuffle. The initiating cause of load reversals is often rapid application or release of the throttle from coast or from drive condition while in low gear and at low road speeds. It can also occur after gear selection and when the clutch is rapidly engaged. Clonk conditions are most noticeable in low gear and while driving at low road speeds. Closely associated with the initial jerk and the clonk response is the subsequent first low-frequency rocking motion of the drivetrain system, known as shuffle, which is accompanied with the fore and aft motions of the vehicle, referred to as shunt [2]. This phenomenon has been investigated numerically by a number of authors for various automotive driveline systems [3-8].

Therefore, low-frequency torsional vibration of the drivetrain system, known as shuffle, and the coupled axial movements of the vehicle, referred to as shunt, initiate the impact of meshing teeth, which initiates the clonk phenomenon. Each cycle of shuffle is accompanied with clonk conditions, appearing at the first swing in rapid torque reversal. Under extreme conditions, repetitive clonk can take place at each cycle of shuffle. The propensity to clonk depends on the extent of backlash in the meshing pairs, which varies from piece to piece depending on the manufacturing processes. Since the impacting energies at any inertia location will be partly radiated as airborne noise, owing to the rapid velocity changes of the moving bodies, and partly dissipated into the nearby resonant structure, it was necessary to consider the frequency domain response at a structural pick-up point.

In order to study the clonk phenomenon, it is clear that a simple model for torsional vibration of the drive system must be employed in order to create driveline shuffle and initiate impact conditions that give rise to a high-frequency clonk response. If low-frequency torsional vibration of the drivetrain system can be minimized, and in particular the peak value of the torque as described above, then the propensity to clonk can be reduced.

Several potential approaches are possible for reducing vibration. In general, these approaches can be classified as:

(a) eliminating the root cause of the vibration (by reducing the gear lash in the driveline);

(b) tuning the system (by optimizing system parameters such as the spring stiffness of the clutch and flywheel inertia);

(c) adding damping to the system (by installing a viscous unit in the driveline); (d) actively controlling the applied load to the system (by dynamically controlling the engine torque).

The perfect approach depends, of course, on the particular driveline. Furthermore, manufacturing and other practical constraints have to be considered when selecting the optimum driveline configurations [9].

Among the numerical optimization algorithms, gradientbased methods have been the most widely used. The optimum obtained from these methods will be a global optimum if the objective and constraints are differentiable and convex [10]. In practice, however, it is very difficult to prove differentiability and convexity. To determine the global optimum, a number of initial points must be checked out to produce a robust solution. In this sense, the gradientbased methods are not robust.

Evolutionary algorithms, genetic algorithms (GAs) in particular, are known to be robust [11] and have been widely used in the field of numerical optimization in recent years. GAs are search algorithms based on the mechanics of natural selection and natural genetics. One of the key features of GAs is that they search from a population of points and not from a single point. In addition, they use objective function information (fitness function) instead of derivatives or other auxiliary knowledge. These features make GAs robust and practical for engineering applications [11].

Although there have been a large number of investigations into the use of evolutionary algorithms in design optimization, few of these have looked at their performance for real vehicular driveline problems. So far, only limited studies have been reported in references [12] to [14], most of these concentrate on the use of more conventional optimization methods. However, GAs have been applied by the present authors in a dynamic model of a driveline system in order to reduce low-frequency torsional vibration [15]. In the present paper, GAs will be used to reduce the high-frequency noise and vibration caused by the clonk phenomenon.

A team of experts initially brainstormed the driveline noise and vibration and identified more than 70 potential causal factors, mainly quantitative [16]. The initial list was reduced to three factors: flywheel inertia, driveline backlash and spring stiffness of the clutch. GAs are shown to be effective and powerful for finding these factors in comparison with other optimization techniques.

\section{MODEL DESCRIPTION}

The purpose in modelling any physical system is to predict and then to optimize the system performance once sufficient confidence with the model has been established. The model should reasonably represent the characteristics of the system under investigation. Figure 1 shows a schematic layout of a complete drivetrain for a conventional rear 


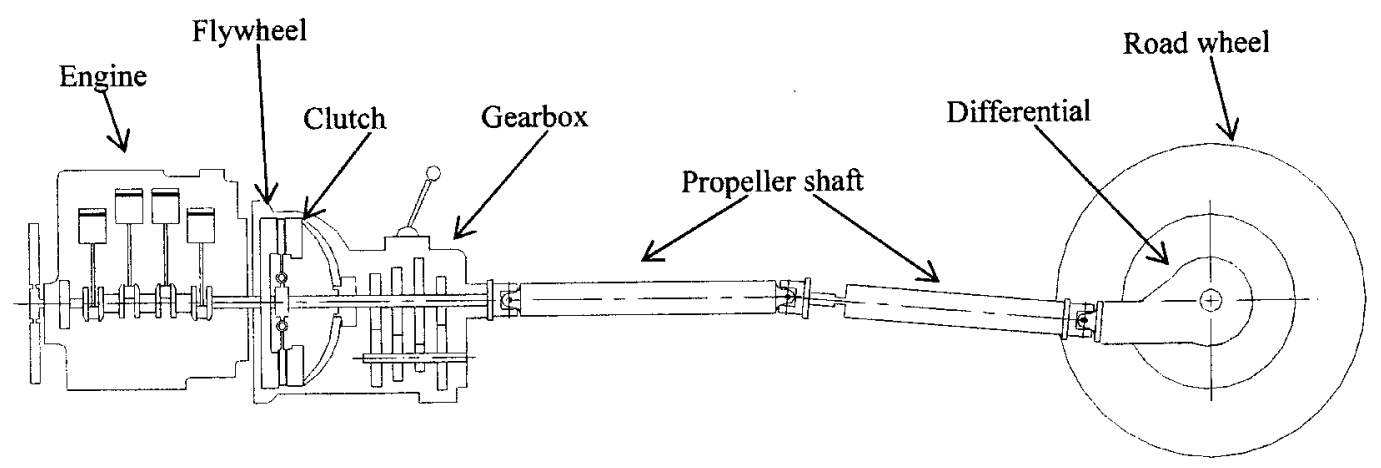

Fig. 1 Classical driveline for a conventional rear wheel drive vehicle

wheel drive vehicle. The distributed-lumped (hybrid) modelling technique (DLMT) is considered for solving the equations of motion.

The driveline consists of two hollow cylindrical driveshafts, both with closed ends, and two solid axle halfshafts. The first driveshaft, as shown in Fig. 2, is $435 \mathrm{~mm}$ long with a constant diameter $(50.7 \mathrm{~mm}$ o.d.). The wall thickness for both the cylinder shell and each of the end walls is $1.65 \mathrm{~mm}$. The second driveshaft is $958 \mathrm{~mm}$ long. The diameter of this tube is not constant along its length. At a distance of $172 \mathrm{~mm}$ from each end, the outside diameter changes from 75 to $90 \mathrm{~mm}$. The axle half-shafts are $877 \mathrm{~mm}$ long and of constant diameter $(30 \mathrm{~mm})$. The distributed components comprise the front and the rear of the driveshaft tubes and the axle half-shafts. On the other hand, the lumped parts of the driveline system are assumed to be the flywheel, clutch, gearbox, differential and road wheels. It is assumed that the rear wheels are fixed to the ground as in the case of the experimental rig described by Menday [16].

\subsection{Distributed shafts}

For the three distributed shafts shown in Fig. 2, the relationship between the angular velocity and the applied torque in the frequency domain can be expressed as [17]

$$
\begin{aligned}
{\left[\begin{array}{l}
T_{2}(\mathrm{i} \omega) \\
T_{3}(\mathrm{i} \omega)
\end{array}\right]=} & {\left[\begin{array}{cc}
\xi_{1} E_{1} & -\xi_{1} \sqrt{\left(E_{1}^{2}-1\right)} \\
\xi_{1} \sqrt{\left(E_{1}^{2}-1\right)} & -\xi_{1} E_{1}
\end{array}\right] } \\
& \times\left[\begin{array}{l}
\omega_{2}(\mathrm{i} \omega) \\
\omega_{3}(\mathrm{i} \omega)
\end{array}\right]
\end{aligned}
$$

for the first shaft of the driveshaft (the impedance module), as

$$
\begin{aligned}
{\left[\begin{array}{l}
\omega_{3}(\mathrm{i} \omega) \\
\omega_{4}(\mathrm{i} \omega)
\end{array}\right]=} & {\left[\begin{array}{cc}
\xi_{2}^{-1} E_{2} & -\xi_{2}^{-1} \sqrt{\left(E_{2}^{2}-1\right)} \\
\xi_{2}^{-1} \sqrt{\left(E_{2}^{2}-1\right)} & -\xi_{2}^{-1} E_{2}
\end{array}\right] } \\
& \times\left[\begin{array}{l}
T_{3}(\mathrm{i} \omega) \\
T_{4}(\mathrm{i} \omega)
\end{array}\right]
\end{aligned}
$$

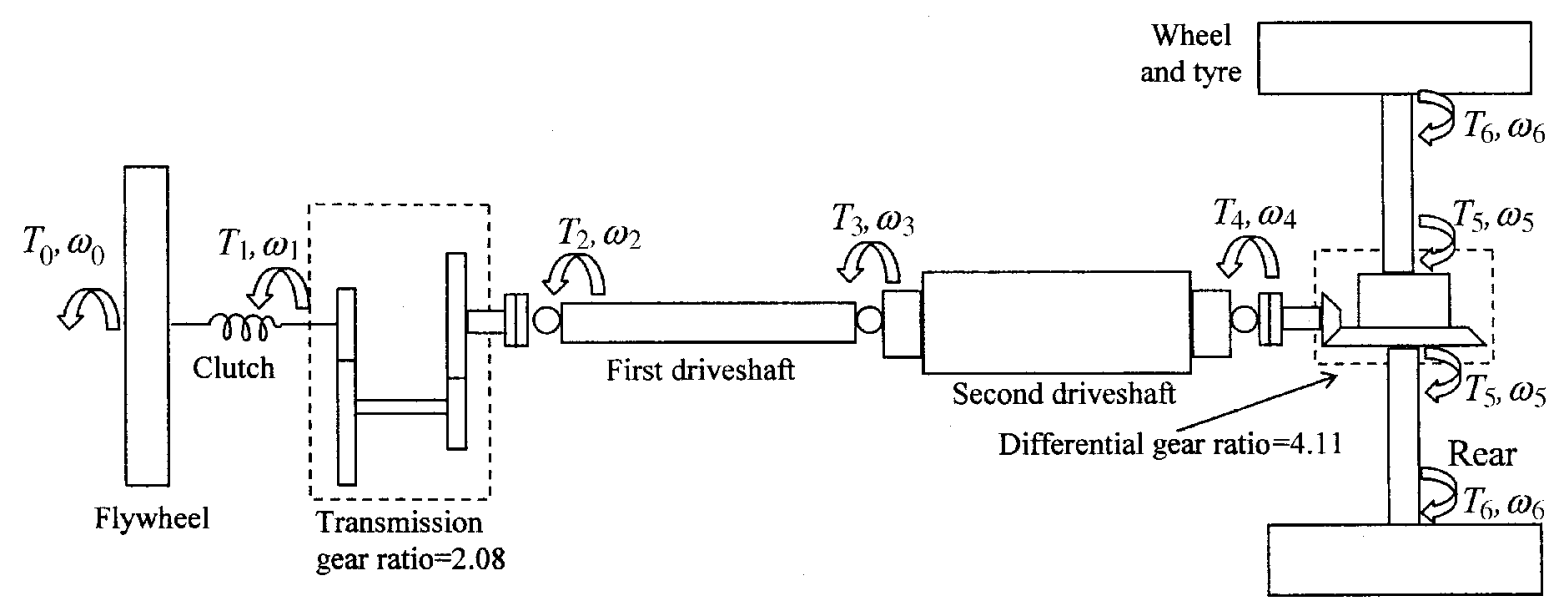

Fig. 2 Distributed-lumped model of the drivetrain system 
for the second shaft of the driveshaft (the admittance module) as

$$
\begin{aligned}
{\left[\begin{array}{l}
T_{5}(\mathrm{i} \omega) \\
T_{6}(\mathrm{i} \omega)
\end{array}\right]=} & {\left[\begin{array}{cc}
\xi_{3} E_{3} & -\xi_{3} \sqrt{\left(E_{3}^{2}-1\right)} \\
\xi_{3} \sqrt{\left(E_{3}^{2}-1\right)} & -\xi_{3} E_{3}
\end{array}\right] } \\
& \times\left[\begin{array}{l}
\omega_{5}(\mathrm{i} \omega) \\
\omega_{6}(\mathrm{i} \omega)
\end{array}\right]
\end{aligned}
$$

for the third distributed axle half-shaft, where

$$
\begin{aligned}
E_{j} & =\frac{e^{\mathrm{i} \omega T_{j \mathrm{~s}}}+1}{e^{\mathrm{i} \omega T_{j \mathrm{~s}}}-1} \\
T_{j \mathrm{~s}} & =2 l_{j} \sqrt{L_{j} C_{j}}=2 l_{j} \sqrt{\frac{\rho_{j}}{G_{j}}} \\
\xi_{j} & =\sqrt{\frac{L_{j}}{C_{j}}}=J_{j} \sqrt{\rho_{j} G_{j}}
\end{aligned}
$$

with $i=1,2,3$. These are the frequency transform variables, the propagation time delays and the characteristic impedances respectively.

\subsection{Lumped flywheel model}

The governing equation for the lumped flywheel, following Laplace transformation with zero initial conditions, is

$$
T_{0}(s)=\left(J_{\mathrm{f}} s+B_{\mathrm{f}}\right) \omega_{0}(s)+T_{1}(s)
$$

By putting $s=\mathrm{i} \omega$ in equation (5), the frequency domain equation can be expressed as

$$
T_{0}(\mathrm{i} \omega)-T_{1}(\mathrm{i} \omega)=\omega_{0}(\mathrm{i} \omega)\left(\mathrm{i} J_{\mathrm{f}} \omega+B_{\mathrm{f}}\right)
$$

\subsection{Lumped clutch model}

The governing equation for the lumped clutch in the Laplace domain is

$$
\begin{aligned}
T_{1}(s) & =\frac{k_{\mathrm{c}}}{s}\left[\omega_{0}(s)-\omega_{1}(s)\right]+C_{\mathrm{c}}\left[\omega_{0}(s)-\omega_{1}(s)\right] \\
& =\left(\frac{k_{\mathrm{c}}}{s}+C_{\mathrm{c}}\right)\left(\omega_{0}-\omega_{1}\right)
\end{aligned}
$$

and in the frequency domain it is

$$
T_{1}(\mathrm{i} \omega)=\left(\frac{-\mathrm{i} k_{\mathrm{c}}}{\omega}+C_{\mathrm{c}}\right)\left[\omega_{0}(\mathrm{i} \omega)-\omega_{1}(\mathrm{i} \omega)\right]
$$

\subsection{Lumped gearbox model}

The governing equations for the lumped gearbox are

$$
\begin{aligned}
& T_{1}(s)=\left(J_{\mathrm{g}} s+B_{\mathrm{g}}\right) \omega_{1}+\frac{T_{2}(s)}{n_{\mathrm{g}}} \\
& \frac{\omega_{1}}{\omega_{2}}=n_{\mathrm{g}}
\end{aligned}
$$

Equation (9) can be expressed in the frequency domain as

$$
T_{1}(\mathrm{i} \omega)-\frac{T_{2}(\mathrm{i} \omega)}{n_{\mathrm{g}}}=\omega_{1}(\mathrm{i} \omega)\left(\mathrm{i} J_{\mathrm{g}} \omega+B_{\mathrm{g}}\right)
$$

\subsection{Lumped differential model}

The governing equation for the lumped differential in the Laplace domain is

$$
\begin{aligned}
& T_{4}(s)=\left(J_{\mathrm{d}} s+B_{\mathrm{d}}\right) \omega_{4}+\frac{T_{5}(s)}{n_{\mathrm{d}}} \\
& \frac{\omega_{4}}{\omega_{5}}=n_{\mathrm{d}}
\end{aligned}
$$

Equation (12) can be expressed in the frequency domain as

$$
T_{4}(\mathrm{i} \omega)-\frac{T_{5}(\mathrm{i} \omega)}{n_{\mathrm{d}}}=\omega_{4}(\mathrm{i} \omega)\left(\mathrm{i} J_{\mathrm{d}} \omega+B_{\mathrm{d}}\right)
$$

\subsection{Lumped road wheel model}

The governing equation for the lumped road wheel model, following Laplace transformation with zero initial conditions, is

$$
T_{6}(s)=\left(J_{\mathrm{w}} s+B_{\mathrm{w}}\right) \omega_{6}(s)
$$

Transforming equation (15) from the $s$ to the $\omega$ domain yields

$$
T_{6}(\mathrm{i} \omega)=\omega_{6}(\mathrm{i} \omega)\left(\mathrm{i} J_{\mathrm{w}} \omega+B_{\mathrm{w}}\right)
$$

\section{DETERMINATION OF THE FREQUENCY RESPONSE}

Using the parameter values given in Table 1, it follows that if the shaft compliance per unit length is given by

$$
\begin{aligned}
& C_{1}=\frac{1}{G_{1} J_{1}}=8.08 \times 10^{-5} 1 / \mathrm{N} \mathrm{m}^{2} \\
& C_{2}=\frac{1}{G_{2} J_{2}}=6.45 \times 10^{-6} 1 / \mathrm{N} \mathrm{m}^{2}
\end{aligned}
$$


Table 1 Driveline parameters

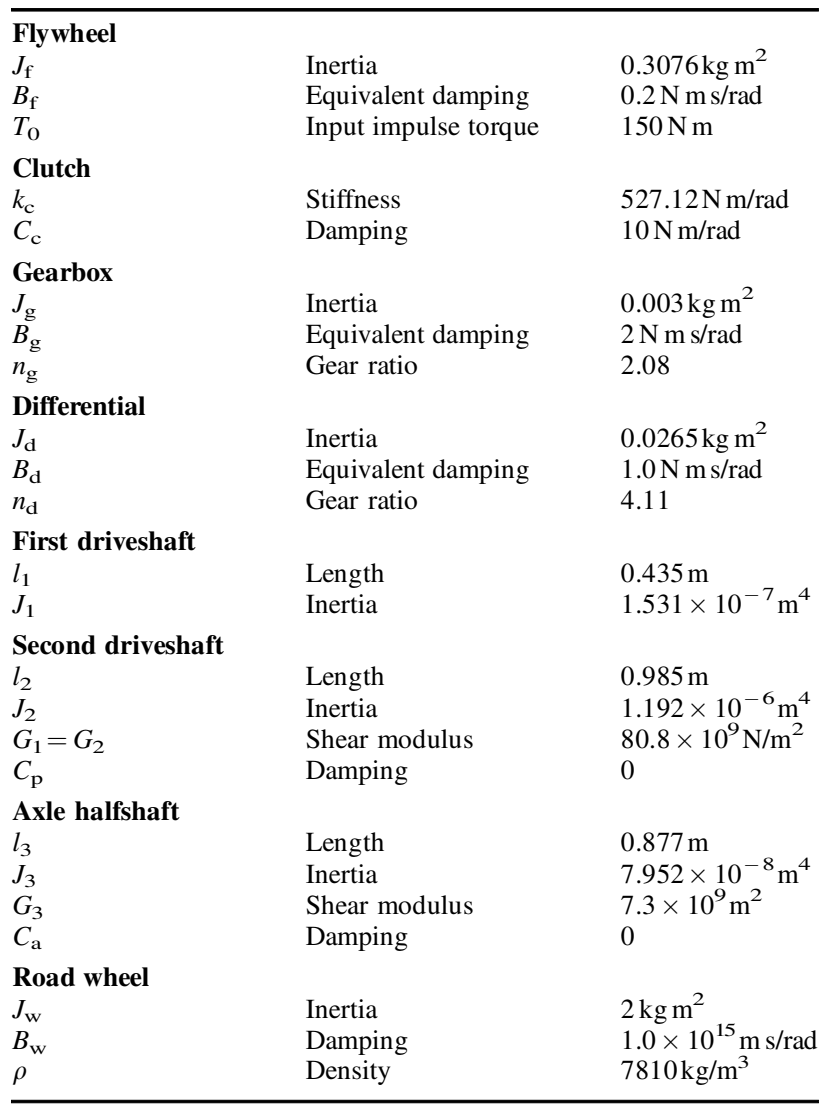

and

$$
C_{3}=\frac{1}{G_{3} J_{3}}=1.62 \times 10^{-4} 1 / \mathrm{N} \mathrm{m}^{2}
$$

and the shaft inertia per metre is given by

$$
\begin{aligned}
& L_{1}=J_{1} \rho=1.19 \times 10^{-3} \\
& L_{2}=J_{2} \rho=14.97 \times 10^{-3}
\end{aligned}
$$

and

$$
L_{3}=J_{3} \rho=6.21 \times 10^{-4}
$$

Then, from equation (4), it follows that

$$
\begin{aligned}
& \xi_{1}=\sqrt{\frac{L_{1}}{C_{1}}}=3.84604 \\
& \xi_{2}=\sqrt{\frac{L_{2}}{C_{2}}}=48.16546
\end{aligned}
$$

and

$$
\xi_{3}=\sqrt{\frac{L_{3}}{C_{3}}}=0.95389
$$

Also

$$
\begin{aligned}
& \frac{T_{\mathrm{p} 1}}{2}=2 l_{1} \sqrt{L_{1} C_{1}}=2.70 \times 10^{-4} \mathrm{~s} \\
& \frac{T_{\mathrm{p} 2}}{2}=2 l_{2} \sqrt{L_{2} C_{2}}=5.95 \times 10^{-4} \mathrm{~s}
\end{aligned}
$$

and

$$
\frac{T_{\mathrm{p} 3}}{2}=2 l_{3} \sqrt{L_{3} C_{3}}=5.57 \times 10^{-4} s
$$

The overall driveline model in DLMT can be represented as a lumped-lumped-lumped-distributed-distributed-lumpeddistributed-lumped (L-L-L-D-D-L-D-L) model, as discussed by Bartlett and Whalley [18]. Since there are three distributed and five lumped parameter sections, using equations (1), (2), (3), (6), (8), (10), (11), (13), (14) and (16), the complete distributed-lumped model of the driveline system can be expressed in matrix form as

$$
\left[\begin{array}{c}
T_{0} \\
0 \\
0 \\
0 \\
0
\end{array}\right]=\left[\begin{array}{ccccc}
a_{11} & a_{12} & 0 & 0 & 0 \\
a_{21} & a_{22} & a_{23} & 0 & 0 \\
0 & a_{32} & a_{33} & a_{34} & 0 \\
0 & a_{42} & a_{43} & a_{44} & a_{45} \\
0 & 0 & 0 & a_{54} & a_{55}
\end{array}\right]\left[\begin{array}{c}
\omega_{0} \\
\omega_{1} \\
\omega_{3} \\
\omega_{4} \\
\omega_{6}
\end{array}\right]
$$

where

$$
a_{11}=\left(B_{\mathrm{f}}+C_{\mathrm{c}}\right)+\mathrm{i}\left(J_{\mathrm{f}} \omega-\frac{k_{\mathrm{c}}}{\omega}\right)
$$

$$
\begin{aligned}
& a_{12}=-C_{\mathrm{c}}+\mathrm{i} \frac{k_{\mathrm{c}}}{\omega} \\
& a_{21}=n_{\mathrm{g}}\left(C_{\mathrm{c}}-\mathrm{i} \frac{k_{\mathrm{c}}}{\omega}\right)
\end{aligned}
$$$$
a_{22}=-n_{\mathrm{g}}\left[C_{\mathrm{c}}+B_{\mathrm{g}}-\xi_{1} \frac{E_{1}}{n_{\mathrm{g}}^{2}}+\mathrm{i}\left(J_{\mathrm{g}} \omega-\frac{k_{\mathrm{c}}}{\omega}\right)\right]
$$$$
a_{23}=\xi_{1} \sqrt{E_{1}^{2}-1}
$$$$
a_{32}=\frac{\xi_{1} \sqrt{E_{1}^{2}-1}}{n_{\mathrm{g}} \xi_{2} E_{2}}
$$$$
a_{33}=-\frac{\xi_{1} E_{1}}{\xi_{2} E_{2}}-1
$$$$
a_{34}=\frac{\sqrt{E_{2}^{2}-1}}{E_{2}}
$$$$
a_{42}=\frac{\xi_{1} \sqrt{\left(E_{1}^{2}-1\right)\left(E_{2}^{2}-1\right)}}{n_{\mathrm{g}} E_{2}}
$$

$a_{43}=\frac{-\xi_{1} E_{1} \sqrt{E_{2}^{2}-1}}{E_{2}}$

$a_{44}=-\left(B_{\mathrm{d}}+\xi_{3} \frac{E_{3}}{n_{\mathrm{d}}^{2}}+\frac{\xi_{2}}{E_{2}}+\mathrm{i} J_{\mathrm{d}} \omega\right)$

$a_{45}=\frac{\xi_{3} \sqrt{E_{3}^{2}-1}}{n_{\mathrm{d}}}$ 


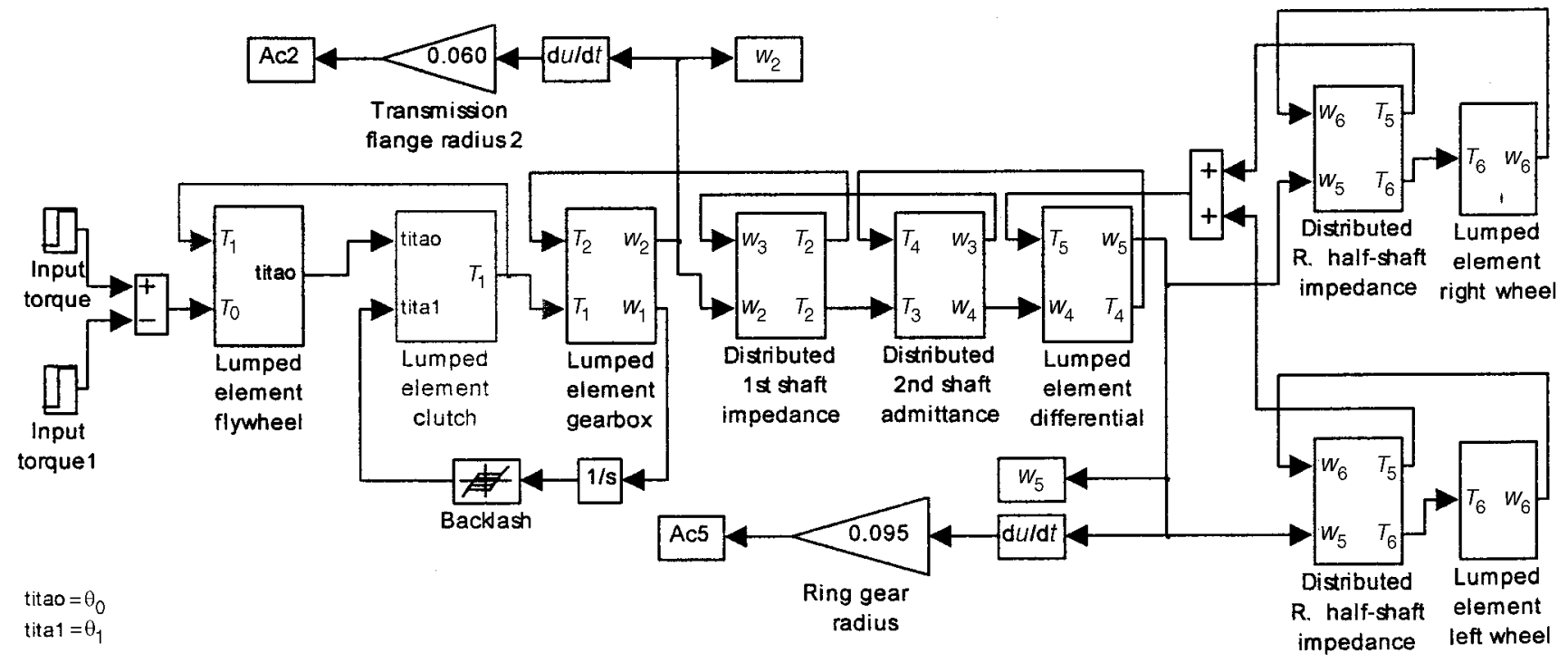

Fig. 3 Driveline as a lumped-lumped-lumped-distributed-distributed-lumped-distributed-lumped (L-L-L-D-D-L-D-L) model

$$
\begin{aligned}
& a_{54}=\frac{\xi_{3} \sqrt{E_{3}^{2}-1}}{n_{\mathrm{d}}} \\
& a_{55}=-\left(B_{\mathrm{w}}+\xi_{3} E_{3}+\mathrm{i} J_{\mathrm{w}} \omega\right)
\end{aligned}
$$

Solving equation (23) for $\omega_{0}, \omega_{1}, \omega_{3}, \omega_{4}$ and $\omega_{6}$ yields

$$
\begin{aligned}
& \frac{\omega_{0}}{T_{0}}(\mathrm{i} \omega)=\frac{\text { numerator }}{\text { denominator }} \\
& \frac{\omega_{1}}{T_{0}}(\mathrm{i} \omega)=\frac{-a_{21}\left(a_{33} a_{45} a_{54}-a_{33} a_{44} a_{55}+a_{34} a_{43} a_{55}\right)}{\text { denominator }} \\
& \frac{\omega_{3}}{T_{0}}(\mathrm{i} \omega)=\frac{a_{21}\left(a_{34} a_{55} a_{42}+a_{32} a_{45} a_{54}+a_{32} a_{44} a_{55}\right)}{\text { denominator }} \\
& \frac{\omega_{4}}{T_{0}}(\mathrm{i} \omega)=\frac{-a_{55} a_{21}\left(a_{42} a_{33}-a_{32} a_{43}\right)}{\text { denominator }} \\
& \frac{\omega_{6}}{T_{0}}(\mathrm{i} \omega)=\frac{a_{54} a_{21}\left(a_{42} a_{33}-a_{32} a_{43}\right)}{\text { denominator }}
\end{aligned}
$$

where

$$
\begin{aligned}
\text { Numerator }= & -a_{23} a_{34} a_{55} a_{42}-a_{33} a_{22} a_{44} a_{55} \\
& +a_{33} a_{54} a_{45} a_{22}+a_{43} a_{34} a_{22} a_{55} \\
& +a_{23} a_{44} a_{32} a_{55}-a_{54} a_{45} a_{23} a_{32} \\
\text { Denominator }= & -a_{11} a_{23} a_{34} a_{55} a_{42}-a_{11} a_{33} a_{22} a_{55} a_{44} \\
& +a_{11} a_{33} a_{54} a_{45} a_{22}+a_{11} a_{43} a_{34} a_{22} a_{55} \\
& +a_{11} a_{23} a_{44} a_{32} a_{55}-a_{11} a_{54} a_{45} a_{23} a_{32} \\
& +a_{33} a_{21} a_{12} a_{44} a_{55}-a_{33} a_{54} a_{45} a_{21} a_{12} \\
& -a_{43} a_{34} a_{21} a_{12} a_{55}
\end{aligned}
$$

Substituting the values for $a_{11}$ to $a_{55}$ into equations (24) to (28), and using equations (17) to (22), the desired frequency response function can be computed.

In this research the DLMT has been used to find the frequency response. It can also be used in the time domain. From the driveline system model, shown in Fig. 2, together with the equations of motion in the time domain, a complete simulation model of the driveline system can be developed, as shown in Fig. 3. This simulation model has been constructed in MATLAB using the SIMULINK toolbox.

\section{EXPERIMENTAL INVESTIGATION OF DRIVELINE NVH}

The rig, shown in Fig. 4, simulates the rear wheel drive drivetrain system of a light truck, consisting of flywheel, clutch, transmission, a two-piece driveshaft assembly, differential and a rear axle assembly comprising rear axle halfshafts, brake assembly and road wheels. The rear wheels were fixed to the ground. A preload torque was applied to the flywheel via a low-inertia disc brake system as stored energy, acting through the torsional clutch springs in the system. This preload was instantaneously released (the action amounting to the application of a Dirac-type function) and was reacted at the fixed rear wheel assemblies. Accelerometer pick-ups were located along the driveline, as shown in Fig. 4, to monitor the response of the system components by subsequent spectral analysis. Time histories at nine accelerometer pick-ups along the driveline (as shown in Fig. 4) were recorded for 16 different configurations at the test rig. Details relating to the experimental rig and the design of experiments are provided by Menday et al. $[4,16]$.

Although the driver requires a responsive vehicle, the impulse may excite an unwanted low-frequency longitudinal 


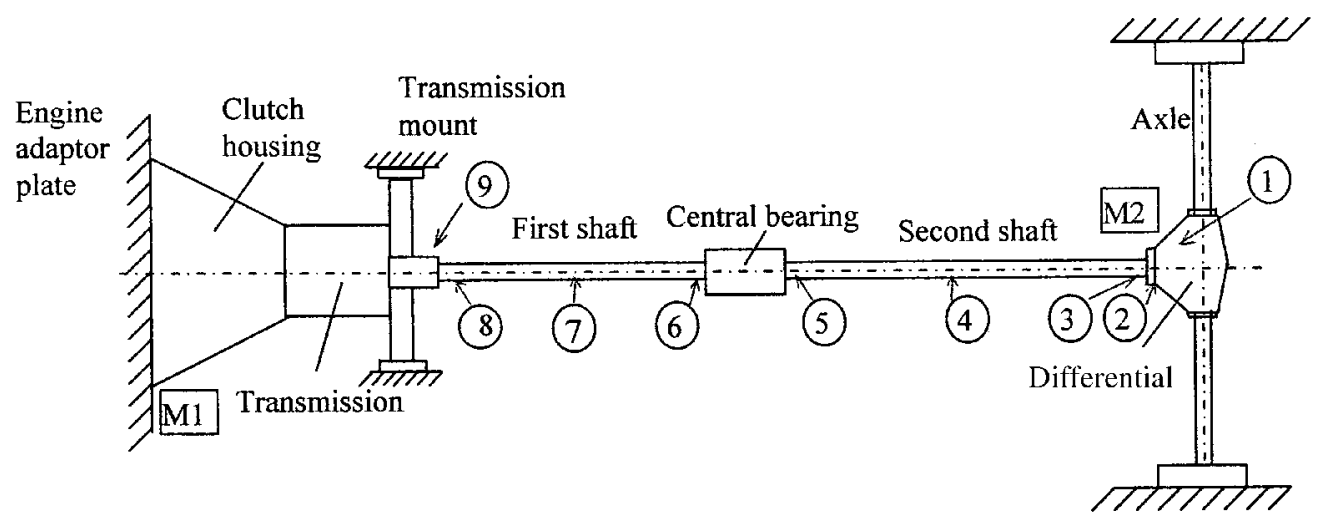

Fig. 4 Schematic representation of the driveline experimental rig: 1, ring gear; 2, axle differential flange; 3 , second driveshaft, differential end location; 4, second driveshaft, centre location; 5, second driveshaft, central bearing location; 6, first driveshaft, central bearing location; 7, first driveshaft, centre location; 8, first driveshaft, transmission end; 9, transmission flange; M1, microphone at transmission bell housing; M2, microphone at axle differential

mode of vibration known as shuffle. Each cycle of the shuffle response may generate clonk noise [2].

\section{GENETIC ALGORITHMS}

A genetic algorithm is a machine learning technique that is based on emulating the principles of natural selection proposed in the Darwinian theory of the natural selection for evolving species. The theory is adapted to artificial scopes, where information is manipulated as a basis for the process of natural selection. Genetic algorithms (GAs) were developed by Holland [19] in 1967 in the University of Michigan, after he had adapted the selection processes of nature to artificial systems, to exploit the advantages of evolution. These algorithms maintain and manipulate a family, or population, of solutions and implement a 'survival of the fittest' strategy in their search for better solutions.

Genetic algorithms require a set of population members, usually numbering between 20 and 100. Each population member represents a trial solution to a given problem. The trial solution-where numbers are used for input variables-is tested by an evaluation function, which calculates the quality of the trial solution. The output is commonly called the fitness, since it describes how fit the trial solution is.

In GAs, the natural parameter set in the optimization problem is coded as a finite-length string. Traditionally, GAs use binary numbers to represent such strings: a string has a finite length and each bit of a string can be either 0 or 1 . For real function optimization, it is more natural to use real numbers. The length of the real-number string corresponds to the number of design variables.

Genetic algorithms commonly work by starting with relatively poor trial solutions, that is, population members with poor fitness. These initial strings are generated randomly. At each generation (iteration) of the GA process, the fitness value (objective function value) of every individual is evaluated and used to specify its probability of reproduction. A new population is generated from selected parents by performing specified operators on their genes. These operators are briefly explained here.

A simple GA is composed of three operators:

(a) selection,

(b) crossover,

(c) mutation.

Selection consists in choosing the solutions that are going to form a new generation. The main idea is that selection should depend on the value of the fitness function: the higher fitness has the higher probability for the individual to be chosen (this is similar to the concept of 'survival of the fittest' in Darwinian theory). However, it remains a probability, which means that it is not a deterministic choice: even solutions with a comparatively low fitness may be chosen, and they may prove to be very good in the course of events (e.g. if the optimization is trapped in a local minimum). The two major selection techniques are roulette wheel and tournament.

The selection process produces a mating pool. Then, crossover proceeds in two steps: members in a mating pool are mated at random, and then, a position (or several) along each pair of strings is selected according to a uniform random law. Finally, based on the crossover probability, $P_{\mathrm{c}}$, the paired strings exchange all characters following the cross-site. This results in a pair of strings of a new generation. Clearly, the crossover randomly exchanges structured information between parents $\mathrm{A}$ and $\mathrm{B}$ to produce two offspring $\mathrm{A}^{\prime}$ and $\mathrm{B}^{\prime}$, which are expected to combine the best characters of their parents as shown in Fig. 5.

The last operator, called mutation, is a random alteration of a bit at a string position and is based on the mutation 


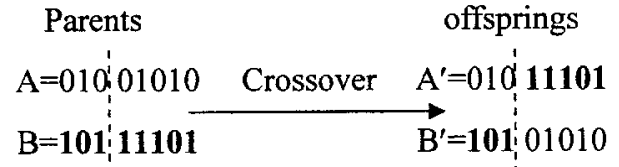

Fig. 5 Crossover process

probability, $P_{\mathrm{m}}$. In the present case, a mutation means changing a bit from 0 to 1 and vice versa. Therefore, mutation is a bit change of a string that occurs during the crossover process at a given mutation rate. Mutation implies a random walk through the string space and plays a secondary role in simple GA. Mutation is important in evolution because, unlike crossover (which merely trades genes), new gene values are introduced. This further increases the diversity of the population members and enables the optimization to get out of local minima.

In a pure (fitness-proportional) selection procedure, each individual in the current population is reproduced a number of times proportional to the individual's performance. Therefore, it does not guarantee the selection of any particular individual, including the fittest. This means that, with this selection technique, the best solution to the problem discovered so far can be regularly thrown away. In an elitist strategy, first pure selection is performed, and then the elitist strategy stipulates that the individual with the best performance always survives intact into the next generation. For many applications the search speed can be greatly improved by not losing the best, or elite, member between generations [20]. Ensuring the propagation of the elite member is termed elitism and requires not only the elite member to be selected but also a copy of it not to become disrupted by crossover or mutation.

At each generation, the population strings are decoded and evaluated in order to measure their fitness value, and then the GA operators described above are applied in order to form the following generation. This process is iterated until convergence is achieved or a near-optimal solution is found. A flow chart of the present GA is illustrated in Fig. 6.

For any GA, a chromosome representation is needed to describe each individual in the population of interest. The representation scheme determines how the problem is structured in the GA and also determines the genetic operators that are used. Each individual or chromosome is made up of a sequence of genes from a certain alphabet. An alphabet could consist of binary digits (0 and 1), floating point number, integer, symbols (i.e. A, B, C, D), matrices, etc. In Holland's original design, the alphabet was limited to binary digits. Since then, problem representation has been the subject of much investigation. It has been shown that more natural representations are more efficient and produce better solutions [21]. One useful representation of an individual or chromosome for function optimization involves genes or variables from an alphabet of floating point numbers with values within variable upper

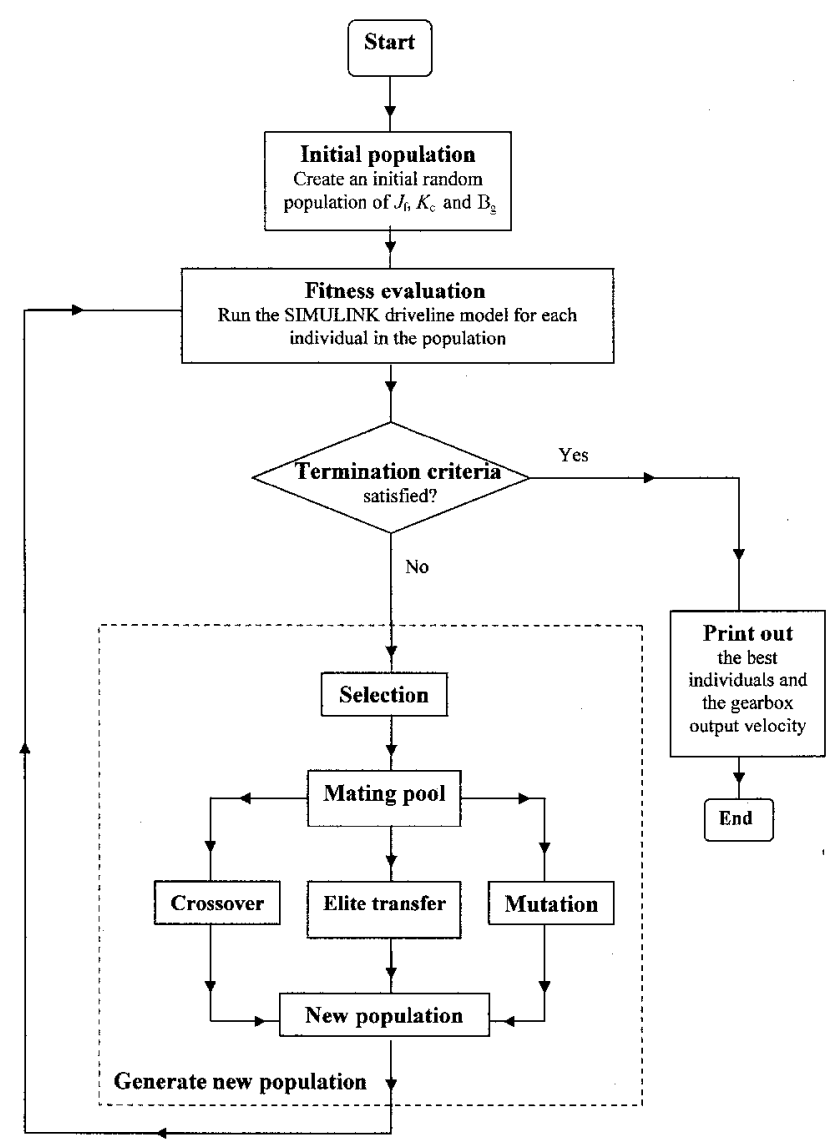

Fig. 6 GA flow chart for driveline optimization

and lower bounds. Michalewicz [21] has done extensive experimentation comparing real-valued and binary GAs and shown that the real-valued GA is an order of magnitude more efficient in terms of CPU time. He has also shown that a real-valued representation moves the problem closer to the problem representation which offers higher precision with more consistent results across replications.

\section{CLONK OPTIMIZATION BY GA}

The purpose of the driveline modelling is to optimize the driveline clonk response to an optimum possible response within realistic constraints. To do this, the parametric studies carried out using the model have two main objectives [9]:

(a) to identify the important subsystems and components affecting clonk,

(b) to specify the value of new test components to be used on the model and the test rig [3].

Based on previous work $[\mathbf{9}, \mathbf{1 3}]$ and preliminary analysis of the model it was established that the more important parameters included torque rise/decay profile and speed, driveline stiffness, driveline inertia, engine mounting system geometry and stiffness, driveline backlash and driveline 
damping. In selecting the bounds of parameter studies it is important to consider what size of parameter changes are realistic. This can sometimes involve special measurements or calculations. For example, a change in driveline backlash can be achieved by modifying many different components and joints between components (such as splines), each of which will have a different contribution to the total backlash, depending on the gear ratio. Also, it is necessary to consider the trade-off with other vehicle attributes such as NVH. For example, gear rattle considerations place a lower bound on flywheel inertia.

From the introduction to the paper it is clear that the initial torque rise rate and backlash are the primary reasons for the severity of the clonk condition. Both these parameters affect the extent of impulsive action, since they determine the impact time (i.e. the impact velocity achieved by motion through backlash). This is analogous to a ball dropping onto a plate, where the impact velocity increases with the height of the ball. Increased inertia of the flywheel reduces torsional vibrations. An increased torsional stiffness of the clutch system, such as when a predamper is used, can have the same desired effect. It is prudent to simplify the analysis by choosing the clutch torsional stiffness the flywheel inertia and the extent of backlash as the parameters to optimize the system dynamics. Therefore, the parameters selected for variation were the flywheel inertia, the driveline backlash and the spring stiffness of the clutch. The original values of the flywheel inertia, the driveline backlash and the spring stiffness of clutch were $0.3076 \mathrm{~kg} \mathrm{~m}^{2}, 0.04 \mathrm{rad}$ and $527.12 \mathrm{Nm} / \mathrm{rad}$ respectively. The use of the simplified drivetrain model described in Section 2 is then justified.

To be able to perform an optimization, it is necessary to find an objective function. The calculation target is to minimize this objective by variation of certain physical parameters (design parameters) within an acceptable range (design space) given by technological restrictions as space or feasibility limitations.

Computationally, any kind of physical quantity (displacement, velocity, acceleration, force, etc.) of the mechanical systems that can be calculated during the simulation process, as well as any physically meaningful combination of them, can serve as a development objective. As a first approach it could be specified as desirable to scan for peak value over a certain time period or to determine transient times or to integrate over an arbitrary function of the physical quantities.

The definition of the objective is the decisive part of the whole optimization procedure. In this problem, minimization of the peak value of the vibration velocity of the gearbox output is considered to be the objective function [17].

\section{RESULTS AND DISCUSSION}

The main objective of this analysis was to study the torsional vibration of the driveline system and to investigate the clonk (high-frequency) phenomenon in order to reduce the noise and vibration. The DLMT was used. This simulation model was constructed in MATLAB using the SIMULINK toolbox. Input values to the driveline model were mainly derived from experimentally measured data or were calculated. The values in Table 1 were substituted into the simulation model in Fig. 3. An impulse torque of $150 \mathrm{~N} \mathrm{~m}$ with a duration time of $40 \mathrm{~ms}$ as input was applied to the flywheel. The experimental results were found to agree with this mathematical model [17].

To undertake the optimization process, two different representation techniques in genetic algorithms, the binary genetic algorithm (BGA) and float genetic algorithm (FGA), were applied. Functionally, both the binary coded and the floating-point coded GA used for experiments were comparable in implementations (both being implemented using MATLAB). The FGA, in comparison with the BGA, has increased power in terms of solution quality and speed of convergence to the optimal solution [15]. The computational overhead due to the coding/decoding of variables to/ from binary representation from/to floating-point representation is quite high, as is the overhead due to the handling of long binary strings. Both are unnecessary operations when floating-point coded GA is used. Since each run of the clonk model takes more than $10 \mathrm{~min}$, owing to the time restriction, the FGA has been chosen to solve the clonk optimization task. For computation, use was made of a single $\mathrm{PC}$ with a $500 \mathrm{MHz}$ Pentium processor in which the computation time of typically around 800 SIMULINK runs was $143.5 \mathrm{~h}$ or about 6 days. With binary coding the computation time was about twice as long, $247 \mathrm{~h}$ or about 12 days.

Using the FGA, with a population size $N=80$ and $g=100$ generations, the obtained results for the clonk optimization task are $J_{\mathrm{f}}=0.4 \mathrm{~kg} \mathrm{~m}^{2}, K_{\mathrm{c}}=400 \mathrm{~N} \mathrm{~m} / \mathrm{rad}$ and $B_{\mathrm{g}}=0.04 \mathrm{rad}$. These results are the same as the shuffle optimization results [15], indicating a strong relation between the audible clonk and the shuffle phenomenon. Clonk may be heard in the first cycle of shuffle response, and the first swing on the transient torque, which is produced by the shuffle phenomenon, is the basis for the clonk response [2]. Therefore, the results obtained were somewhat anticipated, and optimizing the driveline shuffle reduces the driveline clonk as well.

In order to verify the results achieved by applying the FGA, the driveline model was used. Two locations in the driveline system were identified as suitable locations to record data. The first location was at the output shaft of the gearbox. The second location was at the output of the differential. Torsional vibrations of the gearbox input were assumed to be a measure of the noise level of the gearbox. The torsional vibrations of the differential are related to the vibrations of the vehicle body since these vibrations are transmitted to the vehicle body via the rear axles and road wheels. Comparison between the angular velocity of the gearbox and the differential output for the original design and the GA optimized design is shown in 


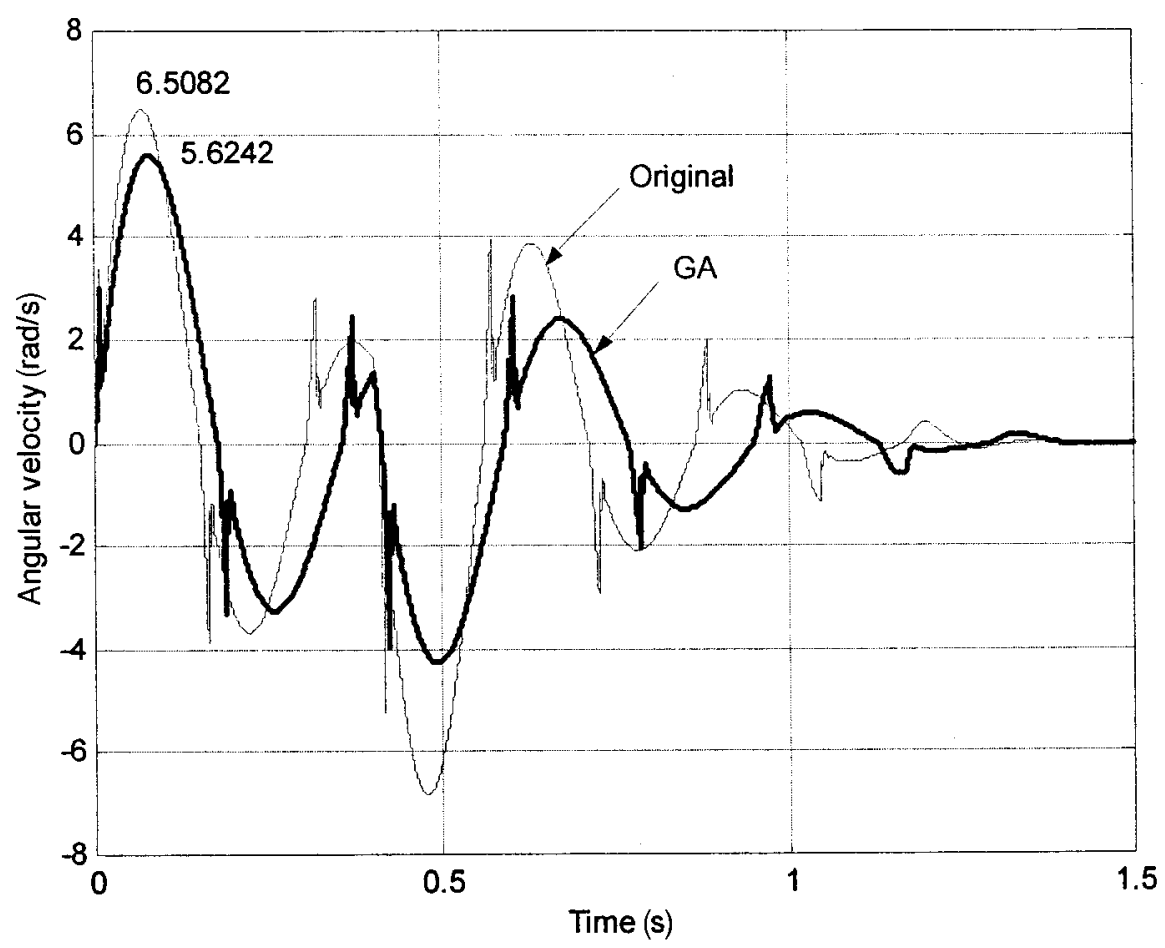

Fig. 7 Comparison between the velocity of the gearbox before and after optimization

Figs 7 and 8 respectively. The FGA reduced the first peak of the angular velocity of the gearbox and the differential output by 13.60 per cent after 800 objective function evaluations. Also, after GA optimization, the acceleration of the gearbox and the differential output were reduced by 16.60 and 19.40 per cent, as shown in Figs 9 and 10 respectively. Figures 11 to 14 show the comparison between the acceleration of the gearbox input, driveshaft,

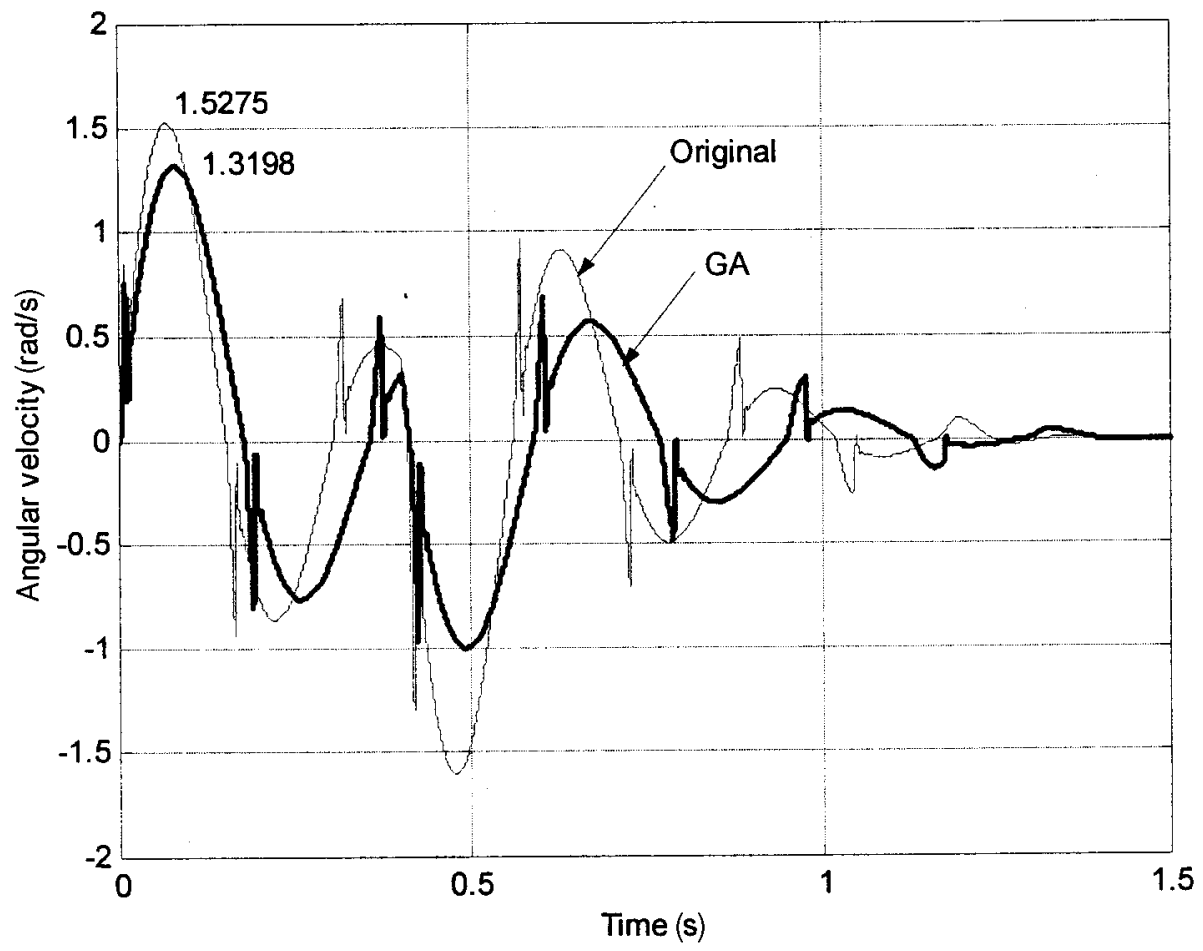

Fig. 8 Comparison between the velocity of the differential before and after optimization 


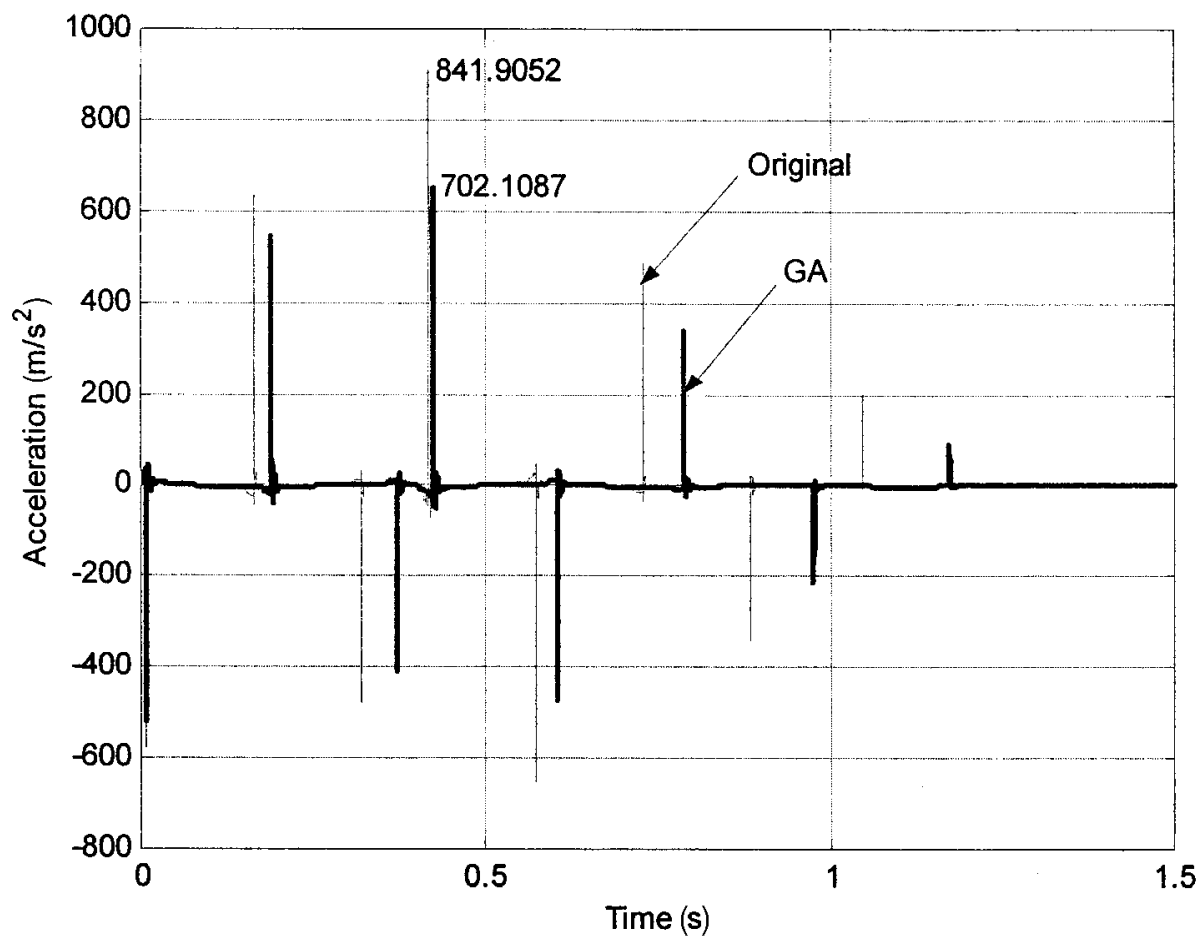

Fig. 9 Comparison between the acceleration of the gearbox before and after optimization

differential input and axle output before and after GA optimization in the frequency domain. In all of the figures the GA shows a significant reduction in the peak value of vibrations. These results indicate the versatility of the present GA approach for design optimization problems in the field of multi-body dynamics. Owing to the large modal density of the drivetrain system, the spectrum of vibration (shown in Figs 11 to 14) contains a multitude of

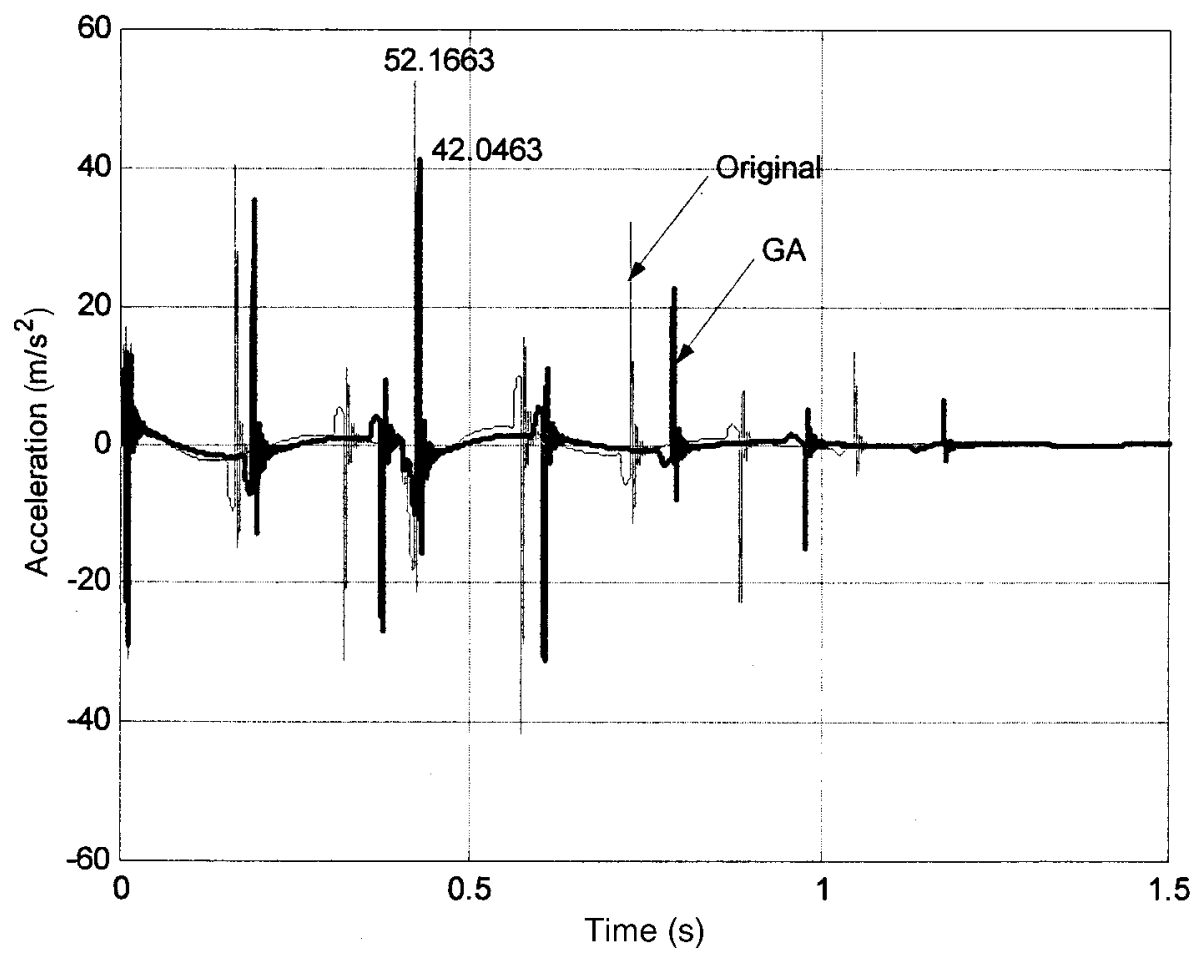

Fig. 10 Comparison between the acceleration of the differential before and after optimization 


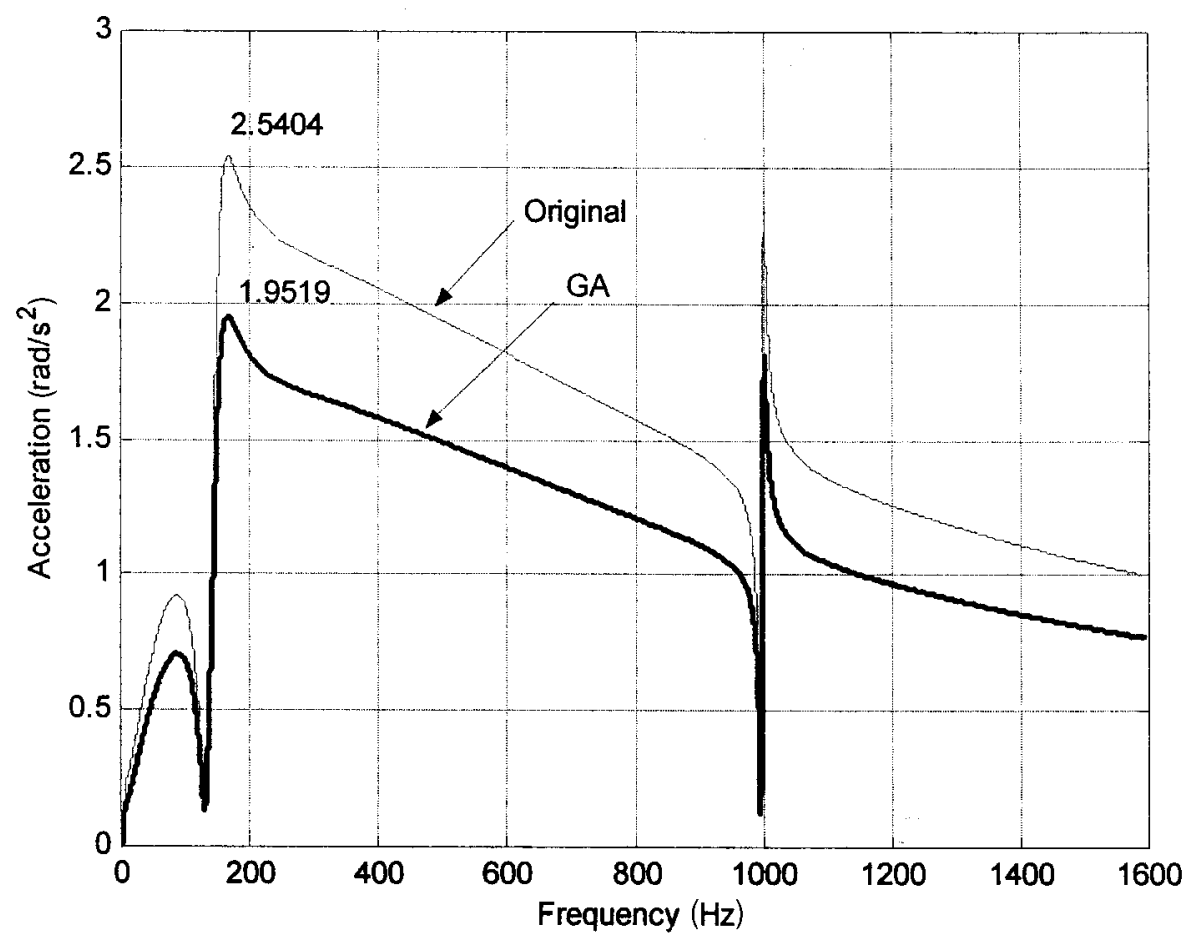

Fig. 11 Comparison between the spectrum of vibration at the gearbox before and after optimization

frequencies. The first $1.51 \mathrm{kHz}$ of the response is shown in the figures, simply to demonstrate the effectiveness of the optimization approach. In view of the simplicity of the model, the specific nature of pronounced peaks cannot be relied upon. However, it is noteworthy that the contribution at $230 \mathrm{~Hz}$ has been observed as the fundamental frequency of hollow driveshaft pieces of the type modelled here [22]. The experimental work in reference

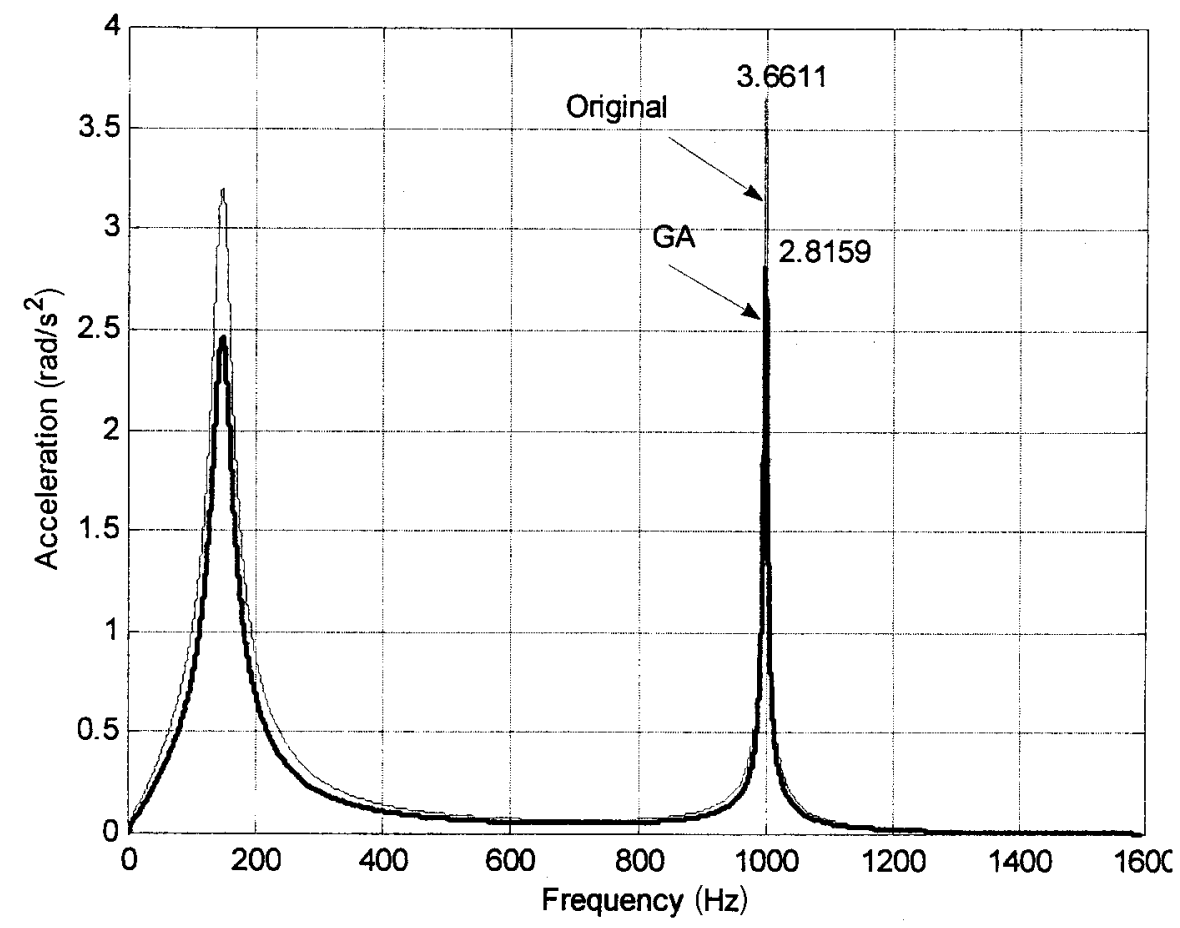

Fig. 12 Comparison between the spectrum of vibration at the driveshaft before and after optimization 


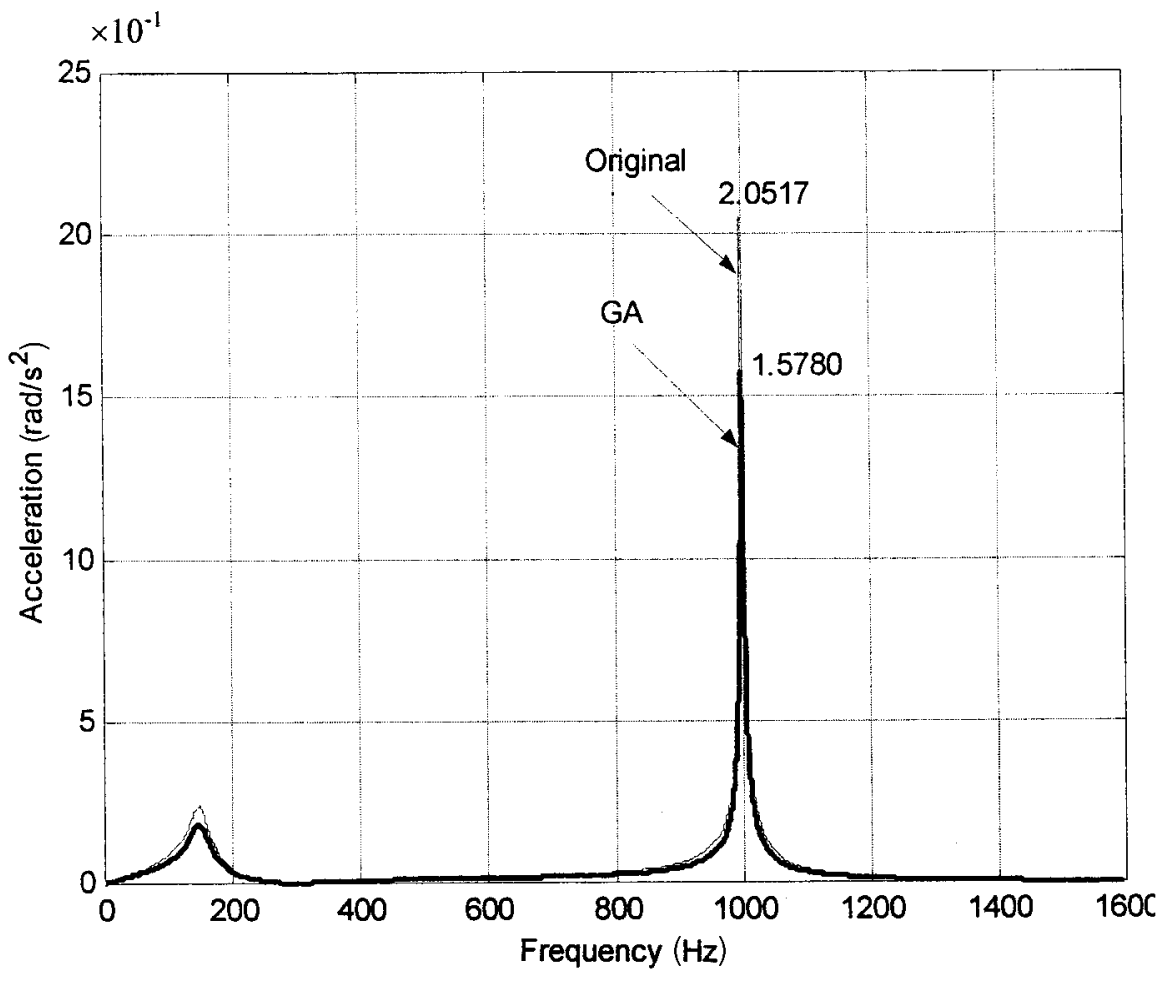

Fig. 13 Comparison between the spectrum of vibration at the differential before and after optimization

[22] identifies the first combined torsion-deflection mode of the system at around $1200 \mathrm{~Hz}$, whereas the work reported in reference [6] isolates a contribution at $1100 \mathrm{~Hz}$ as the structure-acoustic coupling for standing pressure waves inside the front driveshaft tube, using a modified boundary element analysis. Therefore, the contribution at $1000 \mathrm{~Hz}$ obtained in this analysis is quite representative of the real problem.

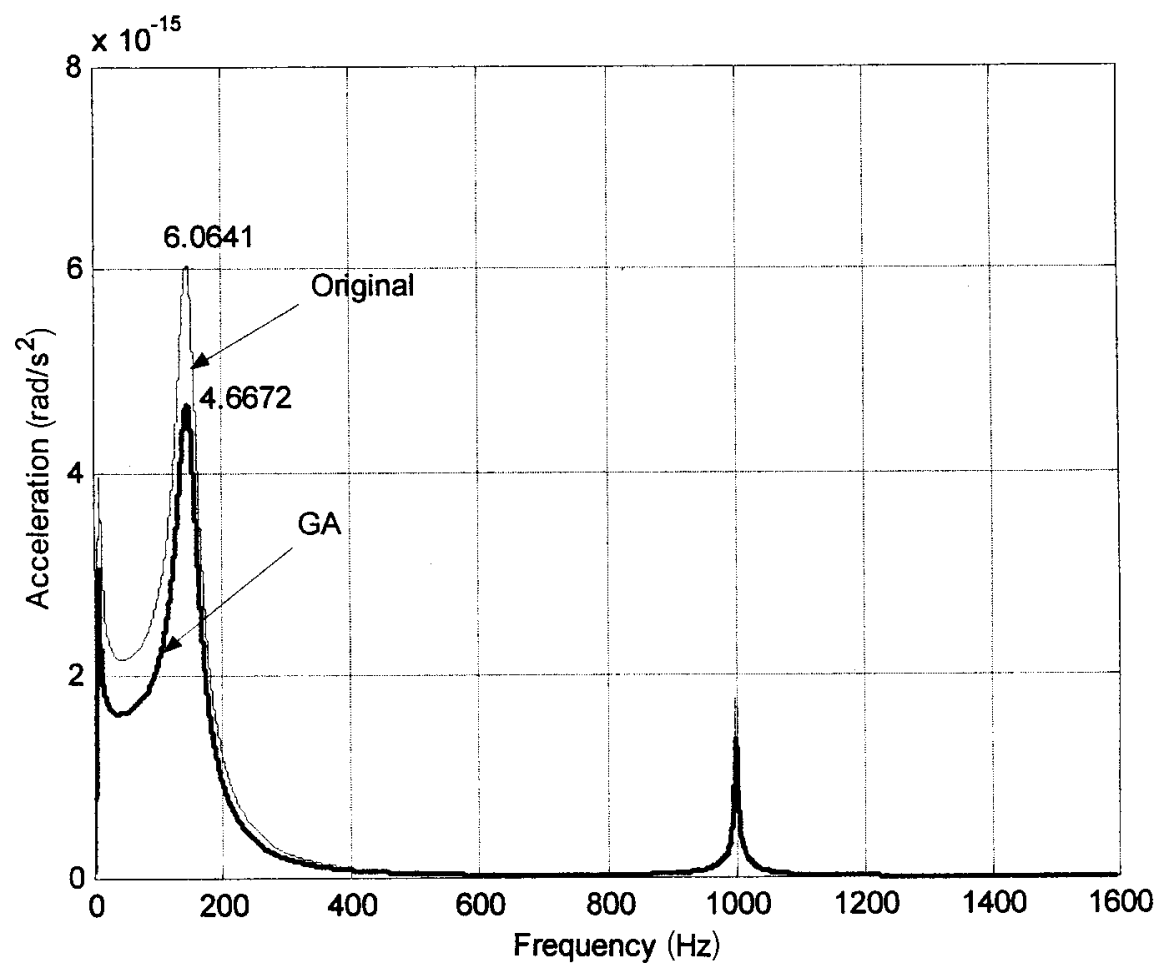

Fig. 14 Comparison between the spectrum of vibration at the rear axle before and after optimization 


\section{CONCLUSION}

This investigation considers the optimization of a vehicular driveline system. The presence of local optima determined the use of a stochastic optimization method to search the design space. The results of this research show that the use of genetic algorithms for function optimization is highly efficient and effective. The effectiveness of the approach depends on a good initial judgement in selection of the parameters to be used in the optimization process. Then, instead of obtaining a unique solution within a domain of possible alternatives, a population of such points is identified, within which the use of an objective function would lead to the optimal solution The advantage of this method is the 'directness' of the search strategy, which leads to a practical approach in identification of the desired goal.

An interesting empirical observation was made when comparing binary and floating-point coded GAs. The total number of individuals needing to be computed to achieve a solution of an acceptable level remains about the same whichever type of GA is used. Owing to the computational overhead for binary representation, the total computational time required for binary coded GA is about twice as long as with floating-point coding.

These results indicate the versatility of the present evolutionary approach for design optimization problems in the field of multi-body dynamics.

\section{ACKNOWLEDGEMENT}

The authors wish to express their gratitude to Ford Motor Company for the financial and technical support extended to this research project. The financial support of the Foresight Vehicle Directorate is also acknowledged

\section{REFERENCES}

1 Rahnejat, H. Multi-body Dynamics Vehicle, Machines and Mechanisms, 1998 (Professional Engineering Publishing, London; Society of Automotive Engineers, Warrendale, Pennsylvania).

2 Krenz, R. Vehicle response to throttle tip-in/tip-out. In Proceedings of Surface Vehicle Noise and Vibration Conference, Michigan, 15-17 May 1985, SAE, Paper 850967, pp. 45-51.

3 Farshidianfar, A., Ebrahimi, M. and Barlett, H. Hybrid modelling and simulation of the torsional vibration of vehicle driveline systems. Proc. Instn Mech. Engrs, Part D, J. Automobile Engineering, 2001, 215, 217-229.

4 Menday, M. T., Rahnejat, H. and Ebrahimi, M. Clonk: an onomatopoeic response in torsional impact of automotive drivelines. Proc. Instn Mech. Engrs, Part D, J. Automobile Engineering, 1999, 213(D4), 349-357.
5 Biermann, J. W. and Hagerodt, B. Investigation of clonk phenomenon in vehicle transmissions-measurement, modelling, and simulation. Proc. Instn Mech. Engrs, Part K, J. Multi-body Dynamics, 1999, 213(J1), pp. 53-60.

6 Arrundale, D., Hussain, K., Rahnejat, H. and Menday, M. T. Acoustic response of driveline pieces under impacting loads (clonk). In Proceedings of 31st ISATA, Dusseldorf, June 1998, pp. 319-331.

7 Arrundale, D., Rahnejat, H. and Menday, M. T. Multi-body dynamics of automobile drivelines: an investigation into the interaction between shuffle and clonk. In Proceedings of 32nd ISATA, Vienna, Austria, June 1999, pp. 397-405.

8 Kelly, P., Menday, M. T. and Rahnejat, H. Powertrain refinement: a combined experimental and multi-body dynamic analysis approach. In Proceedings of 8th Aachen Colloquium, Aachen, Germany, October 1999, pp. 1079-1094.

9 Farshidianfar, A., Ebrahimi, M. Barlett, H. and Moavenian, M. Manufacturing parameter variation in automotive driveline. Advanced Manufacturing Processes, System and Technologies International Conference, 1999, pp. 143-152 (MEP).

10 Vanderplaats, G. N. Numerical Optimization Techniques for Engineering Design: with Applications, 1984 (MCGraw-Hill, New York).

11 Goldberg, D. E. Genetic Algorithms in Search Optimization and Machine Learning, 1989 (Addison-Wesley, Reading, Massachusetts).

12 Hsieh, C. C. and Oh, K. P. A minimization study of automotive driveline vibration. ASME, De-Vol. 40, Advanced Automotive Technologies, 1991, pp. 525-537.

13 Rooke, G. S., Crossley, P. R. and Chan, E. A. Computer modelling of a vehicle powertrain for driveability development. IMechE Report C462/31/035, 1995, pp. 1-9.

14 Stuecklschwaiger, W. and Ronacher, A. W. Optimization of vehicle drive train vibrations using computation techniques. IMechE paper C487/024, 1994, pp. 31-39.

15 Farshidianfar, A. and Ebrahimi, M. Optimization of vehicle driveline vibrations using genetic algorithm (GA). SAE Noise and Vibration Conference and Exposition, Traverse City, Michigan, April 30-May 32001.

16 Menday, M. T. Torsional impact in an automotive vehicle driveline. MSc thesis, University of Bradford, 1997.

17 Farshidianfar, A. Optimisation of torsional vibrations in driveline systems. PhD thesis, University of Bradford, 2001.

18 Barlett, H. and Whalley, R. Analogue solution to the modelling and simulation of distributed-lumped parameter systems. Proc. Instn Mech. Engrs, Part I, J. Systems and Control Engineering, 1998, 212, 99-114.

19 Holland, J. H. Adaptation in Natural and Artificial Systems, 1975 (University of Michigan, Ann Arbor).

20 Coley, D. A. An Introduction to Genetic Algorithms for Scientists and Engineers, 1st edition. 1999 (World Scientific).

21 Michaelwicz, Z. Genetic Algorithms + Data Structures $=$ Evolution Programs, AI Series, 1994 (Springer-Verlag, New York).

22 Vafaei, S., Menday, M. and Rahnejat, H. Transient highfrequency elasto-acoustic response of a vehicular drivetrain to sudden throttle demand. Proc. Instn Mech. Engrs, Part K, J. Multi-body Dynamics, 2001, 215(K1), 35-52. 